This item was submitted to Loughborough's Research Repository by the author.

Items in Figshare are protected by copyright, with all rights reserved, unless otherwise indicated.

\title{
The functional significance of hamstrings composition: is it really a 'fast' muscle group?
}

\section{PLEASE CITE THE PUBLISHED VERSION}

http://dx.doi.org/10.1111/sms.12786

\section{PUBLISHER}

(c) John Wiley \& Sons

\section{VERSION}

AM (Accepted Manuscript)

\section{PUBLISHER STATEMENT}

This work is made available according to the conditions of the Creative Commons Attribution-NonCommercialNoDerivatives 4.0 International (CC BY-NC-ND 4.0) licence. Full details of this licence are available at: https://creativecommons.org/licenses/by-nc-nd/4.0/

\section{LICENCE}

CC BY-NC-ND 4.0

\section{REPOSITORY RECORD}

Evangelidis, Pavlos, Garry J. Massey, Richard Ferguson, Patrick Wheeler, Matthew Pain, and Jonathan Folland. 2016. "The Functional Significance of Hamstrings Composition: Is It Really a 'fast' Muscle Group?". Loughborough University. https://hdl.handle.net/2134/22697. 
1 Title: The functional significance of hamstrings composition: Is it really a 'fast' muscle

2 group?

3 Running head: Is hamstrings really a 'fast' muscle group?

4

5 Authors:

6 Pavlos E. Evangelidis ${ }^{1}$, Garry J. Massey ${ }^{1}$, Richard A. Ferguson ${ }^{1}$, Patrick C. Wheeler ${ }^{1,2}$,

$7 \quad$ Matthew T.G. Pain ${ }^{1}$, Jonathan P. Folland ${ }^{1}$

\section{Affiliations:}

$9{ }^{1}$ School of Sport, Exercise and Health Sciences, Loughborough University, Ashby Road,

10 Loughborough, Leicestershire, LE11 3TU, UK

$11{ }^{2}$ Department of Sport and Exercise Medicine, University Hospitals of Leicester NHS Trust, 12 UK

13 Corresponding author:

14 Pavlos Evangelidis, PhD

15 School of Sport, Exercise and Health Sciences, Loughborough University, Loughborough,

16 LE11 3TU, UK

17 Telephone: $\quad+44(0) 7895563164$

18 Fax: $\quad+44(0) 1509226301$

19 Email: pavlos.evangelidis@gmail.com 


\section{ABSTRACT}

Hamstrings muscle fibre composition may be predominantly fast-twitch and could explain the high incidence of hamstrings strain injuries. However, hamstrings muscle composition in vivo, and its influence on knee flexor muscle function, remains unknown. We investigated biceps femoris long head (BFIh) myosin-heavy chain (MHC) composition from biopsy samples, and the association of hamstrings composition and hamstrings muscle volume (using MRI) with knee flexor maximal and explosive strength. Thirty-one young men performed maximal (concentric, eccentric, isometric) and explosive (isometric) contractions. BFlh exhibited a balanced MHC distribution (mean \pm SD (min-max); 47.1 $19.1 \%$ (32.6-71.0\%)

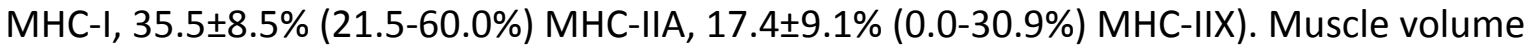
was correlated with knee flexor maximal strength at all velocities and contraction modes ( $r=$ $0.62-0.76, P<0.01$ ), but only associated with late phase explosive strength (time to $90 \mathrm{Nm}$; $r=-0.53, P<0.05)$. In contrast, BFIh muscle composition was not related to any maximal or explosive strength measure. BFIh MHC composition was not found to be 'fast', and therefore composition does not appear to explain the high incidence of hamstrings strain injury. Hamstrings muscle volume explained $38-58 \%$ of the inter-individual differences in knee flexor maximum strength at a range of velocities and contraction modes, while BFIh muscle composition was not associated with maximal or explosive strength.

Keywords: Biceps femoris long head, myosin heavy chain, muscle volume, explosive strength, maximal strength, muscle biopsies, MRI 


\section{INTRODUCTION}

41 The hamstrings muscle group is the primary knee flexor and a major hip extensor and as such the strength of this muscle group is important for human locomotion and athletic activities such as running and jumping. Hamstrings function, particularly rapid 'explosive' force production (i.e. the development of contractile force as quickly as possible from a low or resting level), is also considered important for dynamic knee joint control and stability, and thus maintaining joint integrity (Aagaard et al., 2000; Zebis et al., 2011; Hannah et al., 2014). Furthermore, hamstrings strain injuries are the most common injury in a variety of sprint-based sports (e.g. football and track sprinting; Ekstrand et al., 2011; Alonso et al., 2012) leading to large amounts of time loss in professional and recreational athletes. These injuries most commonly affect the biceps femoris long head muscle (BFlh; Woodley \& Mercer, 2004) and appear to occur during high velocity eccentric muscle actions (e.g. late swing phase of sprinting; Chumanov et al., 2012). Therefore, understanding the function of the hamstrings muscle, including its capacity for maximum strength at a wide range of velocities and contraction modes (i.e. the full torque-velocity relationship) as well as explosive strength, is of considerable interest. However, our understanding of hamstrings morphology (size and composition) and how this relates to contractile function is relatively

57 limited.

For example, hamstrings myosin heavy chain ( $\mathrm{MHC}$ ) composition in young healthy

59 individuals remains unknown, as current BFlh muscle composition data are derived solely from cadavers (Johnson et al., 1973; Garrett et al., 1984; Dahmane et al., 2006). In a much

61 cited study of cadaver specimens, Garret et al. (1984) reported that hamstrings contained a higher proportion of type II fibres than the quadriceps or adductor magnus and suggested 
63 that this muscle composition may contribute to the high susceptibility of the hamstrings to

64 strain injuries. Type II fibres are known to be more susceptible to damage after eccentric

65 contractions (Fridén et al., 1983) and thus suggested to be more vulnerable to strain injury

66 (Brockett et al., 2004). Moreover, the methodological limitations of the study of Garret et al.

67 (1984) (a small sample of 10 elderly cadavers) highlight the need to determine hamstrings

68 muscle composition in healthy young, recreationally active adults in order to understand if

69 the composition of this muscle may contribute to its high incidence of strain injury.

As hamstrings muscle composition has only been determined within cadavers, its influence on muscle function remains unknown. Within the quadriceps femoris, a significant correlation between maximum isometric or isovelocity $\left(15-240^{\circ} \mathrm{s}^{-1}\right)$ strength and composition of the vastus lateralis (VL) has often (Thorstensson et al., 1976; Viitasalo \& Komi, 1978; Aagaard \& Andersen, 1998; Gür et al., 2003) but not always been reported (Inbar et al., 1981; Viitasalo et al., 1981; Schantz et al., 1983). Nevertheless, the balance of evidence from quadriceps studies suggests that hamstrings muscle composition appears likely to influence maximum strength of the knee flexors. Furthermore, in vitro studies demonstrate that type II fibres (MHC-II) have a higher rate of force development (Metzger \& Moss, 1990) and greater maximum force at high velocities of shortening (Bottinelli et al., 1999), yet the influence of MHC composition on in vivo hamstrings function remains

81 unknown.

82 Whilst the influence of hamstrings muscle composition on function in vivo remains to be 83 elucidated, muscle size has been consistently found to be a substantial determinant of 84 isometric strength in various muscles (e.g. elbow flexors, r= 0.81, Erskine et al., 2014; plantar flexors, $r=0.65$, Bamman et al., 2000; knee extensors, $r=0.59$, Maughan et al., 
86 1983). Considering the hamstrings, the four studies we are aware of reported quite diverse

87 relationships between muscle size and isometric/concentric strength measures $(r=-0.22$ to

88 0.80; Kanehisa et al., 1994; Masuda et al., 2003; Akagi et al., 2012; Denadai et al., 2014).

89 However, none of these studies examined eccentric or explosive strength. It is also possible

90 that the combined influence of muscle composition and muscle size may further explain the

91 variability in hamstrings muscle function, although this has not been investigated.

92 Therefore, the aim of this study was to determine the BFlh MHC isoform distribution and to

93 examine the association of hamstrings muscle size and BFIh MHC composition with knee

94 flexor strength, including maximal strength measurements across the torque-velocity

95 relationship (concentric, isometric and eccentric) as well as explosive isometric strength. 
Participants

98 Thirty-one healthy, recreationally active participants (age $21 \pm 3$ y (range: $18-29$ y); height $1.79 \pm 0.07 \mathrm{~m}$; body mass $71.8 \pm 7.3 \mathrm{~kg}$; mean $\pm \mathrm{SD}$ ) took part in this study. Participants had

100 a low to moderate level of physical activity and were not involved in systematic physical training or had any previous experience of strength/power training (i.e. weight training, plyometrics) of the lower body musculature. Their physical activity was assessed with the International Physical Activity Questionnaire (iPAQ) short format (Craig et al., 2003) and their average energy expenditure was $1739 \pm 814$ metabolic equivalent-minutes per week.

105 After completing the physical activity and health screen questionnaires, participants provided written informed consent for their participation in this study, which was approved by the Loughborough University Ethical Advisory Committee. All participants were healthy with no history of musculoskeletal problems or injuries of the lower back, pelvis or legs.

109 Participants were instructed not to take part in any unaccustomed or strenuous physical activity for at least 2 days prior to each laboratory visit and to refrain from alcohol and

111 caffeine for the last $24 \mathrm{~h}$ before each visit.

\section{Overview}

113 Participants visited the laboratory on seven separate occasions, each seven days apart at a consistent time of the day (11:00-16:00 h). All the measurements were conducted on the participants' dominant leg (defined as their kicking leg). The first session involved recording

116 anthropometric data and familiarization with the procedures for testing knee flexor

117 explosive isometric strength that was measured during the second and third sessions. The

118 third and fourth sessions involved familiarization with the isokinetic dynamometer

119 procedures, while the knee flexor torque-velocity relationship was examined in the fifth 
121 assessed by magnetic resonance imaging (MRI) (session 6). In the final session, muscle tissue

122 samples were obtained from the BFlh muscle.

\section{Measurements and Data analysis}

\section{Torque-velocity relationship}

125 The participants were seated on an isokinetic dynamometer chair (Con-Trex MJ, CMV AG,

126 Dübendorf, Switzerland) with a hip angle of $120^{\circ}\left(180^{\circ}=\right.$ full extension $)$. This hip angle is

127 similar to that during late swing phase during sprinting (Thelen et al., 2005). Two 3-point

128 belts secured the torso and additional straps tightly secured the pelvis and the distal thigh

129 of their dominant leg. A brace was also placed in front of the non-involved leg. The

130 alignment of the knee joint centre with the dynamometer rotational axis was performed

131 during isometric knee flexion contractions of $>50 \%$ of maximal isometric voluntary torque at

132 a knee joint angle of $\sim 115^{\circ}$. The dynamometer's shin brace was placed posterior to the

133 shank $\sim 2 \mathrm{~cm}$ above the medial malleolus before the shank was tightly secured to the

134 dynamometer lever arm. The range of motion was established (total: $104^{\circ}$, most flexed-

135 extended crank angle: $67-171^{\circ} ; 180^{\circ}$ full extension) and anatomical zero was set at full

136 extension of the knee joint. Passive torque measurements were recorded while the tested

137 leg was passively moved through the full range of motion and thereafter active torque

138 values were corrected for passive torque. Participants were instructed to grasp the handles

139 next to the seat during maximal contractions. Standardized verbal encouragement was

140 given by the same investigator and online visual feedback of the crank torque was provided

141 on a computer screen. The torque, crank angle and crank velocity signals were sampled at 
$1422000 \mathrm{~Hz}$ with a PC using Spike 2 software (CED, Cambridge, UK) and smoothed with a

143 moving average process over $0.065 \mathrm{~s}$ time epochs before any further analysis.

144 For isometric strength measurement, participants first completed a standardized warm-up

145 consisting of a progressive series of submaximal contractions before they performed two

146 sets of three maximum contractions, one at each of three different crank angles $\left(165^{\circ}, 145^{\circ}\right.$

147 and $125^{\circ}$ in a consistent order; $180^{\circ}=$ full extension) near the angle where knee flexors exert

148 their maximal torque (Knapik et al., 1983). Participants were instructed to flex their knee

149 and "pull" as hard and as fast as possible for 3-5 s. One-minute rest was given between each

150 contraction and 2 min between sets. The contraction with the highest torque irrespective of

151 crank angle was selected for further analysis. Isometric strength was defined as the average

152 torque over a $0.5 \mathrm{~s}$ period around the highest instantaneous torque.

153 For the concentric and eccentric strength measurement, participants first completed a

154 standardized warm-up protocol with five submaximal concentric-eccentric contractions of

155 progressively higher intensity. Then, they performed knee flexors maximal concentriceccentric contractions at $50^{\circ} \mathrm{s}^{-1}$ ( 3 sets of 2 reciprocal contractions) and $350^{\circ} \mathrm{s}^{-1}$ ( 3 sets of 3

157 reciprocal contractions) over the full range of motion. There was $\geq 1$ min rest between each set and $\geq 2$ min rest between velocities. For the concentric-eccentric contractions, the

159 acceleration and deceleration phases were excluded in order to disregard torque overshoot during these phases (Schwartz et al., 2010) and the constant isovelocity period was

161 identified (within $\pm 5 \%$ of the prescribed crank angular velocity). Finally, concentric and eccentric strength at each velocity was defined as the highest instantaneous torque recorded within the isovelocity range of the relevant contractions.

164 The high velocity torque ratio was defined as the concentric strength at $350^{\circ} \mathrm{s}^{-1}$ divided by the isometric strength $\left(T_{\text {con350 }} / T_{\text {isom }}\right)$. 
167 Participants lay in a prone position on a custom-made isometric dynamometer at fixed hip

$168\left(140^{\circ}, 180^{\circ}=\right.$ full extension $)$ and knee $\left(150^{\circ}\right)$ joint angles selected to replicate the joint

169 positions during the late swing phase of sprinting (Thelen et al., 2005) when hamstrings

170 strains are thought to occur. To minimize any extraneous movements, participants were

171 fastened with two straps across the hips, a strap over the lower back and a strap over the

172 distal thigh just above the knee joint. A metal ankle cuff with a lining of high density

173 neoprene was placed $\sim 4 \mathrm{~cm}$ above the medial malleolus and the distal leg was tightly

174 secured to the cuff with straps. Force was measured with a calibrated strain gauge (linear response up to $500 \mathrm{~N}$, Force Logic UK, UK) in series with the ankle cuff and perpendicular to

176 the tibia. The force signal was amplified (x370) and sampled at $2000 \mathrm{~Hz}$ with an external

177 analog-to-digital converter (Micro 1401-3, CED, Cambridge, UK). A PC recorded and

178 displayed the data using the Spike 2 software (CED, Cambridge, UK). The force signal was

179 filtered with a $4^{\text {th }}$ order Butterworth filter with a low pass cut-off frequency of $500 \mathrm{~Hz}$. The

180 distance between the knee joint space and the centre of the ankle cuff was measured to

181 calculate knee flexion torque.

182 After a standardised warm-up, participants performed 3 maximal knee flexion contractions

183 to establish the target torque for the subsequent explosive contractions (see below). A

184 computer screen provided real time visual feedback by displaying the torque response.

185 Thereafter, participants completed 10 explosive contractions with 30 s rest between

186 contractions. They were instructed to contract 'as fast and as hard as possible' for $\sim 1 \mathrm{~s}$ with

187 an emphasis on 'fast' without any countermovement or pre-tension. Real-time visual

188 feedback was provided on the computer screen displaying the torque response, with

189 specific performance feedback of the time from $1 \%$ to $80 \%$ of peak torque. For the 
detection of any countermovement or pre-tension, the resting torque was displayed on a

191 sensitive scale. Standardized verbal encouragement was given throughout the maximal and

192 explosive contractions.

193 During offline analysis, the three valid explosive contractions (achieved torque $\geq 80 \%$ of peak 194 torque with no discernible counter-movement or pre-tension - change of baseline signal

$195<0.2 \mathrm{Nm}$ for the $100 \mathrm{~ms}$ prior to the onset of contraction) with the fastest time from onset to $50 \%$ of peak torque were selected for further analysis. Analysis of these contractions

197 consisted of measurement of the time from contraction onset to 10,50 , and $90 \mathrm{Nm}$ and the time from contraction onset to 15,45 and $75 \%$ of peak torque. We used this approach as it

199 facilitates comparisons over the same range of torques for all participants, and may relate more directly to physiological determinants specific to that range of torques (Maffiuletti et al., 2016). Although this does not directly measure the explosive strength in $\mathrm{Nm} / \mathrm{s}$, it does quantify the explosive strength characteristics. Force onsets were identified manually by visual identification by a trained investigator using a systematic approach which is considered to be more valid than automated methods (Tillin et al., 2013). The three analysed explosive contractions were averaged within each measurement session, before averaging across the two sessions when these measurements were made.

A 1.5 T MRI scanner (Signa HDxt, GE) was used to scan the dominant leg in the supine position with the hip and knee joints extended. T1-weighted axial plane images were acquired from the anterior superior iliac spine to the knee joint space in two overlapping

211 blocks and oil filled capsules were placed on the lateral side of the participants' thigh to help

212 with the alignment of the blocks during analysis. The following imaging parameters were 
used: imaging matrix: $512 \times 512$, field of view: $260 \mathrm{~mm} \times 260 \mathrm{~mm}$, spatial resolution: 0.508

$214 \mathrm{~mm} \times 0.508 \mathrm{~mm}$, slice thickness: $5 \mathrm{~mm}$, inter-slice gap: $0 \mathrm{~mm}$.

215 MR images were analysed with Osirix software (version 4.0, Pixmeo, Geneva, Switzerland).

216 The BFlh, biceps femoris short head, semitendinosus and semimembranosus muscles were

217 manually outlined in every third image starting from the most proximal image in which the

218 muscle appeared. All manual segmentation measurements were completed by the same

219 investigator. Muscle volume was calculated using cubic spline interpolation (GraphPad

220 Prism 6, GraphPad Software, Inc.). To examine reliability of the analysis procedures, the

221 images from 6 randomly selected participants were re-analysed a week later and the coefficient of variation (CV) was calculated. The CV for muscle volume was on average $0.6 \%$.

Muscle samples ( $\sim 0.04 \mathrm{~g}$ ) from the mid-section BFlh ( $\sim 50 \%$ thigh length) of the dominant leg were obtained under local anaesthesia (1\% lidocaine) using the microbiopsy technique (Pro-Mag Ultra, Angiotech, Medical Device Technologies, FL, USA) performed under direct ultrasound guidance. Samples were immediately frozen in liquid nitrogen and stored at $80^{\circ} \mathrm{C}$ for further analysis. MHC content was determined by sodium dodecyl sulphate (SDS) polyacrylamide gel electrophoresis using a method derived from that previously described

230 (Fauteck \& Kandarian, 1995). Electrophoresis (Mini-Protean 3, Bio-Rad) was performed on $6 \%$ (crosslinking $2.7 \%$ ) polyacrylamide resolving gels with $4 \%$ (crosslinking $2.7 \%$ ) stacking gels at $\sim 4^{\circ} \mathrm{C}$. The gels were electrophoresed at a constant $100 \mathrm{~V}$ for $1 \mathrm{~h}$, and thereafter at a constant $6 \mathrm{~mA}$ for $\sim 18 \mathrm{~h}$. Gels were immediately silver stained (SilverQuest Silver Staining

234 Kit, Invitrogen) and protein bands quantified by densitometry (ChemiDoc XRS+ System, Bio- 
236 isoforms: type I, IIA, and IIX (Fig. 1). The MHC analysis was run in duplicate and the mean of

237 the 2 analyses was taken. When the first 2 analyses had a difference $>10 \%$ a third analysis

238 was run. For each individual, the representative MHC distribution was defined as the mean

239 of all repeats in which the different $\mathrm{MHC}$ isoforms were within $10 \%$ between analyses. The

240 CV for repeat samples was 3.9\% for MHC-I, 5.7\% for MHC-IIA and 8.4\% for MHC-IIX.

\section{Statistical analysis}

242 Data are presented as mean \pm SD. One-way analysis of variance was used to examine for

243 differences in muscle volume between the constituents muscles of hamstrings and in knee

244 flexors torque at the different velocities. Bivariate relationships were examined using

245 Pearson product moment correlations between the dependent variables and the Holm-

246 Bonferroni correction was used to control for multiple tests. The level of significance was set

247 at $P<0.05$. All statistical procedures were performed with IBM SPSS 22 (IBM Corporation,

248 Armonk, NY). 


\section{RESULTS}

250

Descriptive data on BFlh MHC isoform distribution, hamstrings muscle size and knee flexor

251 strength

252 On average, the BFlh muscle exhibited a balanced, mixed MHC distribution with $47.1 \pm 9.1 \%$ MHC-I, $35.5 \pm 8.5 \%$ MHC-IIA and $17.4 \pm 9.1 \%$ MHC-IIX, but with considerable variation

254 between individuals (Table 1 and Fig. 2). Total hamstrings muscle volume was on average $255794.1 \pm 122.2 \mathrm{~cm}^{3}(\mathrm{CV}=15.4 \%)$, while the BFIh had smaller volume $\left(210.0 \pm 37.9 \mathrm{~cm}^{3}\right)$ than 256 the other biarticular muscles (ST; $228.6 \pm 45.4 \mathrm{~cm}^{3}, P<0.05$ and SM; $234.8 \pm 47.7 \mathrm{~cm}^{3}, P<$ $257 \quad 0.01$, Table 1).

258 The knee flexors exerted their highest torque during slow eccentric contractions (131.1 \pm 27.4 Nm), and there was considerable inter-individual variability at all contraction modes

260 and velocities $(C V=16.9-22.3 \%$, Table 1$)$. The high velocity torque ratio $\left(T_{\text {con350 }} / T_{\text {isom }}\right)$ was 261 $0.51 \pm 0.10(C V=18.6 \%)$. Knee flexor explosive strength characteristics, measured as time to specific torques, were found to vary between individuals, particularly during the later stages of the explosive contractions (Fig. 3).

Relationships of hamstrings muscle size and BFIh MHC isoform distribution with knee flexion strength all velocities ( $r=0.62-0.76, P<0.01$, Table 2$)$. In contrast, no relationship was found between BFlh muscle composition and maximal strength at any velocity $(-0.22<r<0.20, P>0.05$, Fig. 4) or $T_{\text {con350 }} / T_{\text {isom }}(-0.16<r<0.24, P>0.05)$. When torque values at all velocities were 
expressed relative to muscle volume there remained no association with BFIh muscle

271 composition $(-0.29<r<0.35, P>0.05)$.

272 Hamstrings muscle volume was unrelated to explosive strength characteristics (Table 3),

273 measured as time to achieve low absolute levels or relative measures of torque, however it

274 was associated with explosive strength characteristics (time) to high absolute levels of

275 torque (time to $90 \mathrm{Nm}$; $\mathrm{r}=-0.53, P<0.05$ ). BFlh $\mathrm{MHC}$ distribution was unrelated to any

276 measure of explosive strength $(-0.20<r<0.24, P>0.05$, Table 3$)$. 


\section{DISCUSSION}

279 This study examined the influence of hamstrings muscle size and BFlh muscle composition

280 on knee flexors maximal and explosive strength. We found that within the examined cohort, the BFIh exhibited on average a balanced $\mathrm{MHC}$ isoform distribution that appears comparable to that of the vastus lateralis within the quadriceps (see below), and therefore does not support the suggestion that the high incidence of strain injury in this muscle is a result of BFIh composition. Further, we found that $38-58 \%$ of the variance in knee flexor maximum torque at isometric and at a range of concentric and eccentric velocities was attributable to differences in hamstrings muscle volume, while BFlh MHC distribution was not related to any measure of maximal or explosive strength.

The present study is the first to directly examine the BFlh muscle composition in vivo and our results showed that, on average, the BFlh muscle had a balanced distribution of slow and fast MHC isoforms (47.1 $\pm 9.1 \% \mathrm{MHC}-\mathrm{I}$ and $52.9 \pm 9.1 \%$ total MHC-II) in young healthy men. In a much cited study, Garret et al. (1984) reported a BFlh muscle composition within a small cohort of elderly cadavers to be similar to our data $(54.5 \pm 2.8 \%$ type II fibres and 45.5 $\pm 2.8 \%$ type I of total number of sampled fibres). Nevertheless, based on the small differences they observed between the hamstrings and other muscles (quadriceps, 51.9\%; adductor magnus, 44.8\% type II fibres) Garrett et al. (1984) argued that the 'high proportion' of fast fibres in the hamstrings may contribute to their susceptibility to injury. However, our in vivo hamstrings muscle composition data do not support this proposition when compared to equivalent VL data. For example, Staron et al. (2000) reported the VL to contain a greater proportion of MHC-II isoform (66.1\% total MHC-II in 95 physically active young men) compared to the BFlh in the current study. Nevertheless, other studies have 
reported a more balanced VL MHC distribution $(n=28,49 \pm 18 \%$ MHC-I, $35 \pm 16 \%$ MHC-IIA, $16 \pm 10 \%$ MHC-IIX; Taylor et al., 1997). Based on our findings, the BFlh does not have a 'fast' composition, and appears to have a MHC distribution similar or slower than the VL.

Consequently, the composition of the BFlh does not seem to explain the high incidence of strain injuries within this muscle compared to other muscles. Therefore, other aspects of hamstrings structure (e.g. aponeurosis size; Evangelidis et al., 2015) or function (eccentric actions at long lengths; Thelen et al., 2005) are likely to explain the high incidence of strain injuries in this muscle. On an individual basis however, it is possible that the proportion of MHC-II isoforms could still be a risk factor for hamstrings strain injury. Type II fibres are more susceptible to eccentric exercise-induced muscle damage (Fridén et al., 1983), possibly due to structural differences between fibre types (e.g. thinner Z-disks in type II fibres; Fridén \& Lieber, 1992). The accumulation of microscopic eccentric exercise-induced muscle damage and subsequent changes in function (reduction of force-generating capacity, shift of optimum fibre length and impairment of the excitation-contraction coupling; Morgan \& Allen, 1999) could contribute to a macroscopic injury (Brockett et al., 2004). Within our cohort, total MHC-II isoform content ranged from $29.0-67.4 \%$ and it is possible that individuals with a high proportion of type II fibres could be at higher risk of injury. Future retrospective and prospective studies are needed to elucidate the relationship between muscle composition and the incidence of individual strain injuries.

Whilst MHC composition is a strong determinant of contractile function in single fibres

321 (Metzger \& Moss, 1990; Bottinelli et al., 1999), in this study no correlation was found between BFlh muscle composition and knee flexors maximal or explosive strength in vivo. 
our findings; however similar studies on knee extensors reported mixed results for the relationship of maximum/explosive strength with muscle composition. Some of the studies on knee extensors examined these relationships with participants from diverse training and athletic backgrounds e.g. untrained, endurance, and strength and power athletes (Viitasalo \& Komi, 1978; Viitasalo et al., 1981; Gür et al., 2003). Whilst this approach produces a wide range of muscle composition values, numerous other neuromuscular characteristics also likely vary between these groups (e.g. muscle size, architecture, neural drive) and these could confound any relationship of maximum/explosive strength and muscle composition. In the present study within a group of non-athletic young men, muscle composition did not explain their differences in maximal or explosive strength despite the large inter-individual variability in these measures (CV; maximal strength: 16.9-22.3\%; explosive strength: 15.744.1\%). Whilst theoretically it may be surprising that muscle composition was not more strongly predictive of function, this may reflect the extensive range of factors that influence in-vivo function; most obviously muscle size (discussed below), but also including muscle moment arm, neuromuscular activation of the agonists and antagonists, fibre length and pennation, specific tension, parallel and series connective tissue (Folland \& Williams, 2007). Inter-individual differences in these factors may oppose or supersede any functional differences due to muscle composition and thus conceal its contribution to maximal or explosive strength in vivo. For example, agonist neural activation seems to be the primary determinant of voluntary explosive contractions, particularly during the initial phase of contraction ( $<75 \mathrm{~ms}$ ), explaining up to $83 \%$ of the differences within healthy individuals (de Ruiter et al., 2006, 2007; Klass et al., 2008; Folland et al., 2014). However, when neural drive is controlled - via electrical stimulation - the differences in MHC content (Harridge et al., 
1996) or intrinsic contractile properties, that reflect muscle composition, become more evident (Andersen \& Aagaard, 2006; Folland et al,. 2014).

349 Our results revealed that hamstrings volume explained a significant portion of the variance 350 in isometric (38\%), concentric (50-55\%) and eccentric (48-58\%) knee flexor strength. These values are within the range of previous reports of hamstrings isometric/concentric strength (Kanehisa et al., 1994; Masuda et al., 2003; Akagi et al., 2012). The importance of muscle size for eccentric hamstrings strength is a more novel finding. Considering that hamstrings strain injury appears to predominantly occur during eccentric contractions (Chumanov et al., 2012), this finding supports the notion that strength training with an emphasis on hypertrophic adaptations may be a valid approach for preventing hamstrings injury. The proportion of the variance in knee flexor strength explained by hamstrings size may be partly due to the fact that other muscles (i.e. gastrocnemius, gracilis, sartorius and popliteus) contribute to knee flexor torque in addition to the hamstrings, but also the extensive range of neuromuscular factors found to influence function (discussed above). Overall our finding that muscle size explains a substantial proportion of the variance in maximum strength, but composition does not account for any variance, is similar to observations made in two small studies $(n<16)$ in the knee extensors (Maughan \& Nimmo, 1984; Johansson et al., 1987).

In contrast to maximal strength, explosive strength was not influenced by muscle size apart from at high levels of absolute torque (time from rest to $90 \mathrm{Nm} ; \mathrm{r}=-0.53, P<0.01$ ). Whilst no similar data exist on hamstrings, elbow flexor explosive isometric strength has been related to muscle volume during a similar late phase of contraction (150 ms, $r=0.69, P<0.001$; 
production appears to be predominantly explained by agonist neural activation and the

371 contractile (twitch) response to a single action potential, together explaining $77 \%$ of the

372 variance in force after $50 \mathrm{~ms}$ of contraction (Folland et al., 2014).

373 In conclusion, the balanced MHC distribution found in BFlh muscle appears to be

374 comparable or slower to that of the $\mathrm{VL}$, and therefore seems unlikely to contribute to the

375 high susceptibility of the BFlh to strain injury. Hamstrings muscle volume explained $38-58 \%$

376 of the inter-individual differences in knee flexors torque at a range of velocities and

377 contraction modes, while BFlh muscle composition was not associated with maximal or

378 explosive strength.

\section{Perspectives}

380 Our data show that, within recreationally active young men, hamstrings exhibit a balanced

381 muscle composition that seems to be comparable to that of the VL. Based on these findings,

382 the suggestion that hamstrings contain primarily fast-twitch fibres and that this muscle

383 composition may explain the high rates of hamstrings strain injuries was not supported.

384 From a functional perspective, muscle composition was not a determinant of knee flexor

385 function. In contrast, muscle size explained a large proportion of knee flexor maximal

386 strength and a moderate proportion of late phase explosive strength. 


\section{REFERENCES}

388 Aagaard P, Andersen JL. Correlation between contractile strength and myosin heavy chain

389 isoform composition in human skeletal muscle. Med Sci Sports Exerc 1998: 30: 1217-1222.

390 Aagaard P, Simonsen EB, Andersen JL, Magnusson SP, Bojsen-M øller F, Dyhre-Poulsen P.

391 Antagonist muscle coactivation during isokinetic knee extension. Scand J Med Sci Sports

392 2000: 10: 58-67.

393 Akagi R, Tohdoh Y, Takahashi H. Muscle strength and size balances between reciprocal

394 muscle groups in the thigh and lower leg for young men. Int J Sports Med 2012: 33: 386-389.

395 Alonso JM, Edouard P, Fischetto G, Adams B, Depiesse F, Mountjoy M. Determination of

396 future prevention strategies in elite track and field: analysis of Daegu 2011 IAAF

397 Championships injuries and illnesses surveillance. Br J Sports Med 2012: 46: 505-514.

398 Bamman MM, Newcomer BR, Larson-Meyer DE, Weinsier RL, Hunter GR. Evaluation of the

399 strength-size relationship in vivo using various muscle size indices. Med Sci Sports Exerc

$400 \quad 2000: 32: 1307-1313$.

401 Bottinelli R, Pellegrino MA, Canepari M, Rossi R, Reggiani C. Specific contributions of various 402 muscle fibre types to human muscle performance: an in vitro study. J Electromyogr Kinesiol 403 1999: 9: 87-95.

404 Brockett CL, Morgan DL, Proske U. Predicting hamstring strain injury in elite athletes. Med 405 Sci Sports Exerc 2004: 36: 379-387.

406 Chumanov ES, Schache AG, Heiderscheit BC, Thelen DG. Hamstrings are most susceptible to 407 injury during the late swing phase of sprinting. Br J Sports Med 2012: 46: 90. 

and validity. Med Sci Sports Exerc 2003: 35: 1381-1395.

411 Dahmane R, Djordjevic S, Smerdu V. Adaptive potential of human biceps femoris muscle

412 demonstrated by histochemical, immunohistochemical and mechanomyographical

413 methods. Med Biol Eng Comput 2006: 44: 999-1006.

414 de Ruiter CJ, Van Leeuwen D, Heijblom A, Bobbert MF, de Haan A. Fast unilateral isometric 415 knee extension torque development and bilateral jump height. Med Sci Sports Exerc 2006: 416 38: 1843-1852.

417 de Ruiter CJ, Vermeulen G, Toussaint HM, de Haan A. Isometric knee-extensor torque 418 development and jump height in volleyball players. Med Sci Sports Exerc 2007: 39: 13364191346.

420 Denadai BS, de Oliveira FB, Camarda SR, Ribeiro L, Greco CC. Hamstrings-to-quadriceps 421 strength and size ratios of male professional soccer players with muscle imbalance. Clin 422 Physiol Funct Imaging 2014 Oct 27 doi: 10.1111/cpf.12209; Epub ahead of print.

423 Ekstrand J, Hägglund M, Waldén M. Epidemiology of muscle injuries in professional football 424 (soccer). Am J Sports Med 2011: 39: 1226-1232.

425 Erskine RM, Fletcher G, Folland JP. The contribution of muscle hypertrophy to strength 426 changes following resistance training. Eur J Appl Physiol 2014: 114: 1239-1249.

427 Evangelidis PE, Massey GJ, Pain MT, Folland JP. Biceps Femoris Aponeurosis Size: A Potential 428 Risk Factor for Strain Injury? Med Sci Sports Exerc 2015: 47: 1383-1389. 
429 Fauteck SP, Kandarian SC. Sensitive detection of myosin heavy chain composition in skeletal 430 muscle under different loading conditions. Am J Physiol 1995: 268: C419-424.

431 Folland JP, Buckthorpe MW, Hannah R. Human capacity for explosive force production:

432 neural and contractile determinants. Scand J Med Sci Sports 2014: 24: 894-906.

433 Folland JP, Williams AG. The adaptations to strength training: morphological and

434 neurological contributions to increased strength. Sports Med 2007: 37: 145-168.

435 Fridén J, Lieber RL. Structural and mechanical basis of exercise-induced muscle injury. Med 436 Sci Sports Exerc 1992: 24: 521-530.

437 Fridén J, Sjöström M, Ekblom B. Myofibrillar damage following intense eccentric exercise in 438 man. Int J Sports Med 1983: 4: 170-176.

439 Garrett WE Jr, Califf JC, Bassett FH 3rd. Histochemical correlates of hamstring injuries. Am J $440 \quad$ Sports Med 1984: 12: 98-103.

441 Gür H, Gransberg L, vanDyke D, Knutsson E, Larsson L. Relationship between in vivo muscle 442 force at different speeds of isokinetic movements and myosin isoform expression in men 443 and women. Eur J Appl Physiol 2003: 88: 487-496.

444 Hannah R, Minshull C, Smith SL, Folland JP. Longer electromechanical delay impairs

445 hamstrings explosive force versus quadriceps. Med Sci Sports Exerc 2014: 46: 963-972.

446 Inbar O, Kaiser P, Tesch P. Relationships between leg muscle fiber type distribution and leg 447 exercise performance. Int J Sports Med 1981: 2: 154-159. 

marathon runners. Does isokinetic knee extensor performance reflect muscle size and

450 structure? Acta Physiol Scand 1987: 130: 663-669.

451 Johnson MA, Polgar J, Weightman D, Appleton D. Data on the distribution of fibre types in 452 thirty-six human muscles. An autopsy study. J Neurol Sci 1973: 18: 111-129.

453 Kanehisa H, Ikegawa S, Fukunaga T. Comparison of muscle cross-sectional area and strength 454 between untrained women and men. Eur J Appl Physiol 1994: 68: 148-154.

455 Klass M, Baudry S, Duchateau J. Age-related decline in rate of torque development is 456 accompanied by lower maximal motor unit discharge frequency during fast contractions. J 457 Appl Physiol 2008: 104: 739-746.

458 Knapik JJ, Wright JE, Mawdsley RH, Braun J. Isometric, isotonic, and isokinetic torque 459 variations in four muscle groups through a range of joint motion. Phys Ther 1983: 63: 938460947.

461 Maffiuletti NA, Aagaard P, Blazevich AJ, Folland J, Tillin N, Duchateau J. Rate of force 462 development: physiological and methodological considerations. Eur J Appl Physiol 2016: doi 463 10.1007/s00421-016-3346-6 [Epub ahead of print]

464 Masuda K, Kikuhara N, Takahashi H, Yamanaka K. The relationship between muscle cross465 sectional area and strength in various isokinetic movements among soccer players. J Sports 466 Sci 2003: 21: 851-858.

467 Maughan RJ, Nimmo MA. The influence of variations in muscle fibre composition on muscle 468 strength and cross-sectional area in untrained males. J Physiol 1984: 351: 299-311. 
469 Maughan RJ, Watson JS, Weir J. Strength and cross-sectional area of human skeletal muscle.

470 J Physiol 1983: 338: 37-49.

471 Metzger JM, Moss RL. Calcium-sensitive cross-bridge transitions in mammalian fast and slow

472 skeletal muscle fibers. Science 1990: 247: 1088-1090.

473 Morgan DL, Allen DG. Early events in stretch-induced muscle damage. J Appl Physiol 1999:

474 87: 2007-2015.

475 Schantz P, Randall-Fox E, Hutchison W, Tydén A, Astrand PO. Muscle fibre type distribution, 476 muscle cross-sectional area and maximal voluntary strength in humans. Acta Physiol Scand $477 \quad$ 1983: 117: 219-226.

478 Schwartz FP, Bottaro M, Celes RS, Brown LE, Nascimento FA. The influence of velocity 479 overshoot movement artifact on isokinetic knee extension tests. J Sports Sci Med 2010: 9: $480 \quad 140-146$.

481 Staron RS, Hagerman FC, Hikida RS, Murray TF, Hostler DP, Crill MT, Ragg KE, Toma K. Fiber 482 type composition of the vastus lateralis muscle of young men and women. J Histochem 483 Cytochem 2000: 48: 623-629.

484 Taylor AD, Humphries B, Smith P \& Bronks R (1997). Electrophoretic separation of myosin 485 heavy chain isoforms in the human m. vastus lateralis: references to reproducibility and 486 relationships with force, electromechanical delay, fibre conduction velocity, endurance and 487 electromyography. Arch Physiol Biochem 105, 10-18. 
488 Thelen DG, Chumanov ES, Hoerth DM, Best TM, Swanson SC, Li L, Young M, Heiderscheit BC.

489 Hamstring muscle kinematics during treadmill sprinting. Med Sci Sports Exerc 2005: 37: 108-

490114.

491 Thorstensson A, Grimby G, Karlsson J. Force-velocity relations and fiber composition in

492 human knee extensor muscles. J Appl Physiol 1976: 40: 12-16.

493 Tillin NA, Pain MT, Folland JP. Identification of contraction onset during explosive

494 contractions. Response to Thompson et al. "Consistency of rapid muscle force

495 characteristics: influence of muscle contraction onset detection methodology" [J

496 Electromyogr Kinesiol 2012;22(6):893-900]. J Electromyogr Kinesiol 2013: 23: 991-994.

497 Viitasalo JT, Hakkinen K, Komi PV. Isometric and dynamic force production and muscle fibre 498 composition in man. J Hum Mov Stud 1981: 7: 199-209.

499 Viitasalo JT, Komi PV. Force-time characteristics and fiber composition in human leg

500 extensor muscles. Eur J Appl Physiol 1978: 40: 7-15.

501 Woodley SJ, Mercer SR. Hamstring strains - where do they occur? N Z J Physiother 2004: 32:

$502 \quad 22-28$.

503 Zebis MK, Andersen LL, Ellingsgaard H, Aagaard P. Rapid hamstring/quadriceps force

504 capacity in male vs. female elite soccer players. J Strength Cond Res 2011: 25: 1989-1993. 


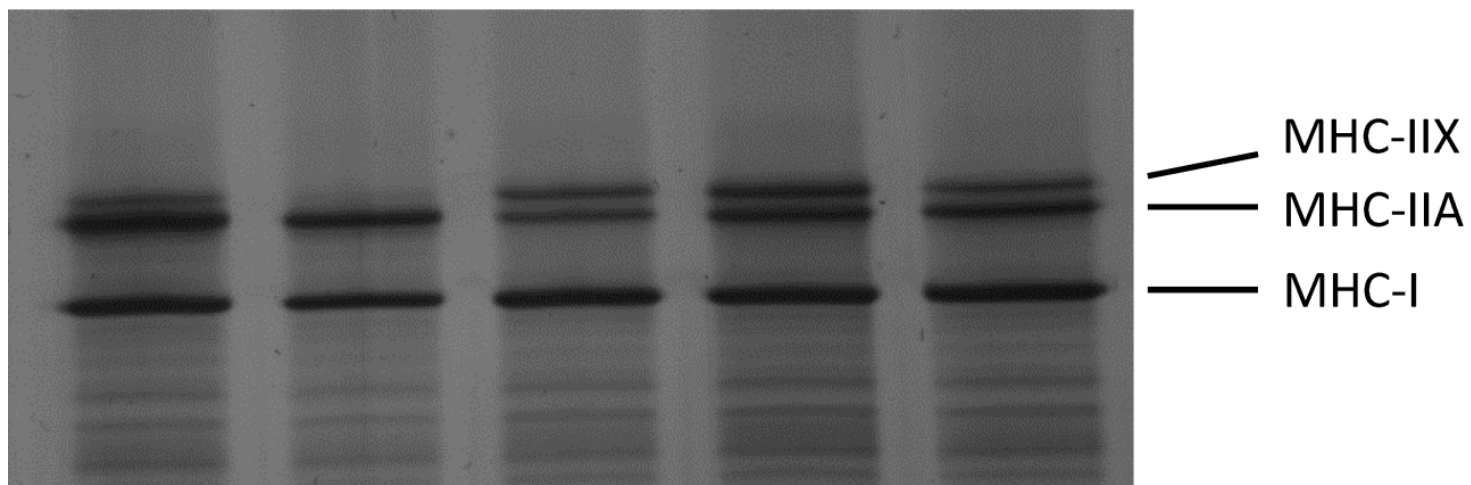

508 Figure 1. Example sodium dodecyl sulphate (SDS) polyacrylamide gel electrophoresis

509 separation of the different myosin heavy chain (MHC) isoforms in biceps femoris long head

510 muscle sampled from 5 participants. 


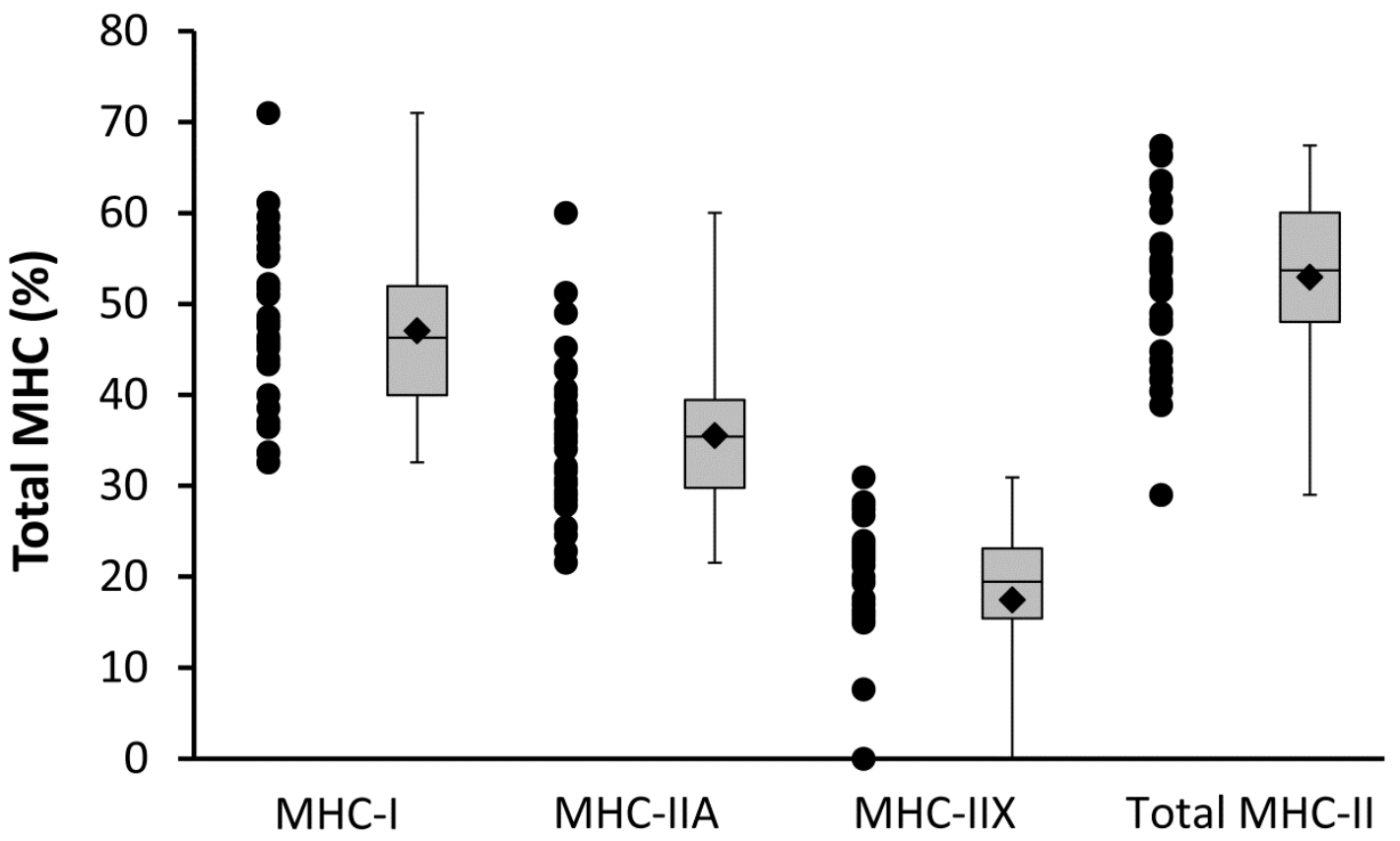

514 Figure 2. BFlh MHC isoform relative distribution $(n=31)$ presented in a combined box plot

515 and scatterplot. Box plot's whiskers correspond to minimum and maximum values and the

516 filled rhombi correspond to the group mean values. 

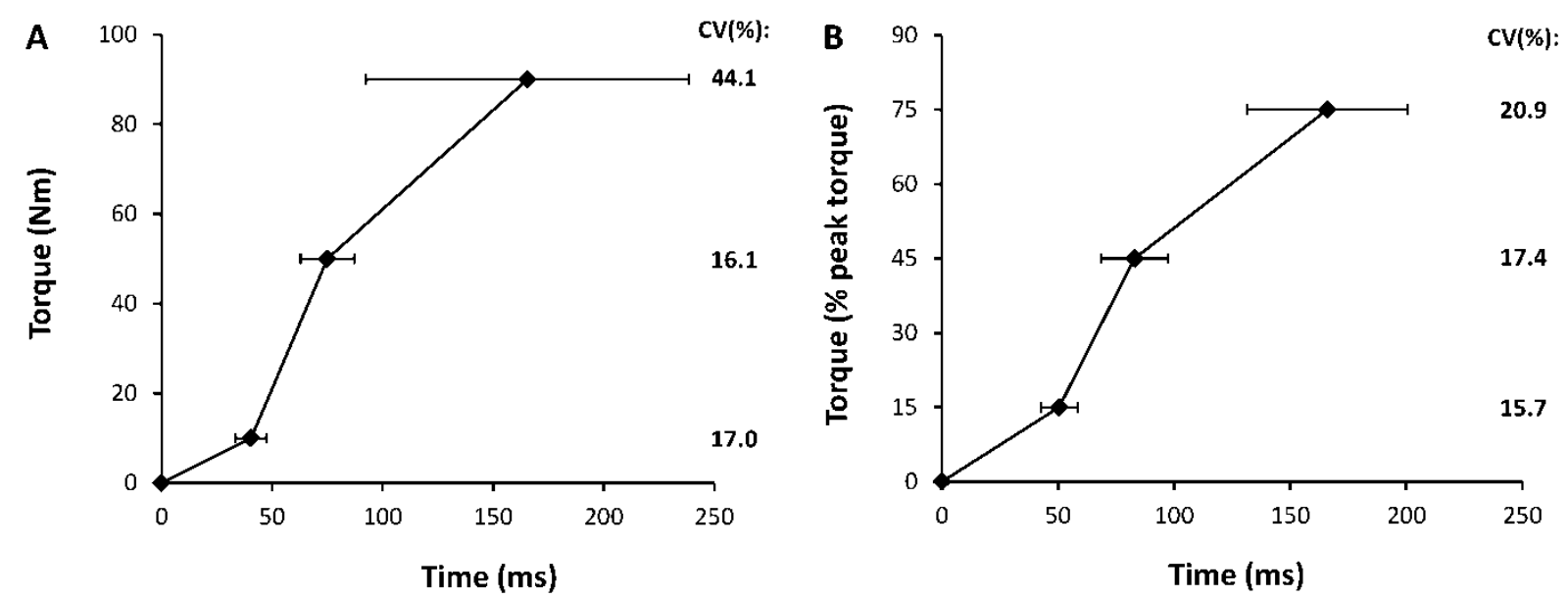

519 Figure 3. Knee flexion explosive strength characteristics expressed as time from zero to

520 absolute (A) and relative (B) torque levels. Data expressed as mean $\pm S D(n=31)$ with inter-

521 individual coefficient of variation (CV) presented at each torque level. 

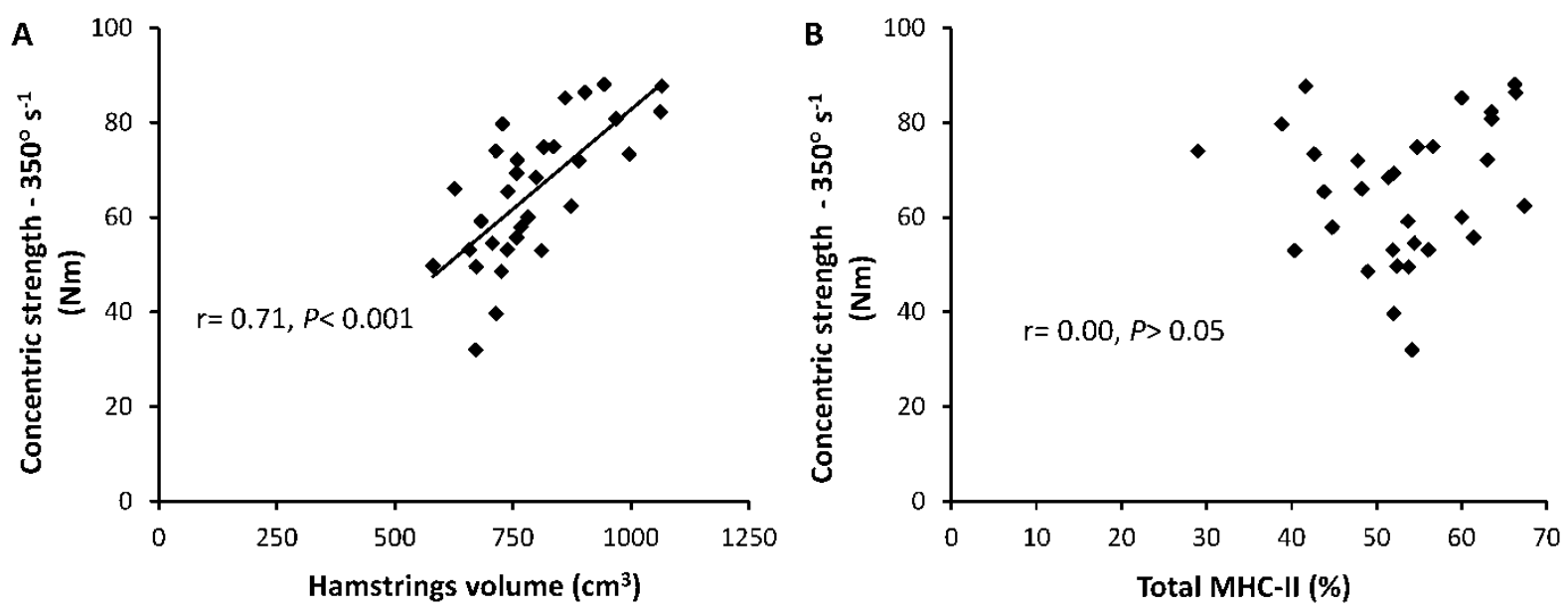

524 Figure 4. Relationships between concentric strength at $350^{\circ} \mathrm{s}^{-1}$ and $(\mathrm{A})$ hamstrings volume

525 and (B) BFlh total MHC-II isoform content ( $n=31)$. BFlh: biceps femoris long head, MHC:

526 myosin heavy chain. 
529 Table 1. Descriptive data of biceps femoris long head muscle composition, hamstrings

530 muscle volume, and knee flexor strength.

\begin{tabular}{|c|c|c|c|c|}
\hline & & Mean \pm SD & Range & CV (\%) \\
\hline Muscle & MHC-I & $47.1 \pm 9.1$ & $32.6-71.0$ & 19.3 \\
\hline \multirow[t]{2}{*}{ composition (\%) } & MHC-IIA & $35.5 \pm 8.5$ & $21.5-60.0$ & 23.9 \\
\hline & MHC-IIX & $17.4 \pm 9.1$ & $0.0-30.9$ & 52.4 \\
\hline \multirow{5}{*}{$\begin{array}{l}\text { Muscle } \\
\left(\mathrm{cm}^{3}\right)\end{array}$} & BFlh & $210.0 \pm 37.9$ & $157.4-289.4$ & 18.1 \\
\hline & BFsh & $120.6 \pm 22.3^{\dagger}$ & $76.6-170.4$ & 18.5 \\
\hline & ST & $228.6 \pm 45.4^{*}$ & $120.5-342.5$ & 19.9 \\
\hline & SM & $234.8 \pm 47.7^{\dagger}$ & $125.3-330.2$ & 20.3 \\
\hline & Total & $794.1 \pm 122.2$ & $581.1-1065.6$ & 15.4 \\
\hline \multirow[t]{6}{*}{ Maximal strength } & Ecc $350^{\circ} \mathrm{s}^{-1}$ & $115.7 \pm 23.6^{\dagger}$ & $58.0-164.5$ & 20.4 \\
\hline & Ecc $50^{\circ} s^{-1}$ & $131.1 \pm 27.4$ & $66.9-174.2$ & 20.9 \\
\hline & Isometric & $128.3 \pm 21.7$ & $92.1-176.4$ & 16.9 \\
\hline & Con $50^{\circ} \mathrm{s}^{-1}$ & $108.3 \pm 21.1^{\dagger}$ & $65.9-154.0$ & 19.5 \\
\hline & Con $350^{\circ} \mathrm{s}^{-1}$ & $65.4 \pm 14.6^{\dagger}$ & $32.0-88.1$ & 22.3 \\
\hline & $\mathbf{T}_{\text {con350 }} / \mathbf{T}_{\text {isom }}$ & $0.51 \pm 0.10$ & $0.29-0.71$ & 18.6 \\
\hline \multicolumn{5}{|c|}{ Data presented as mean \pm SD $(n=31)$. The muscle volumes of the constituent muscles we } \\
\hline
\end{tabular}


534 ST, semitendinosus; SM, semimembranosus; Ecc, eccentric; Con, concentric; ${ }^{*} P<0.05,{ }^{\dagger} P<$ $535 \quad 0.01$.

536 
537 Table 2. Bivariate correlation coefficients of knee flexor maximal strength with hamstrings 538 muscle volume and biceps femoris long head muscle composition

\section{BFlh muscle composition}

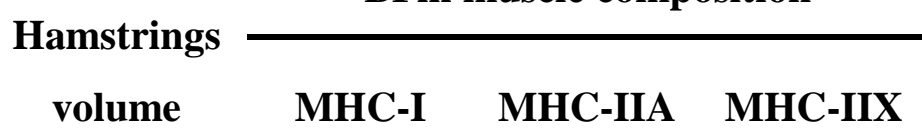

\begin{tabular}{|c|c|c|c|c|c|}
\hline \multirow[t]{6}{*}{ Maximal strength } & Isometric & $0.62^{\dagger}$ & 0.01 & 0.20 & -0.20 \\
\hline & $\operatorname{Con} 50^{\circ} \mathrm{s}^{-1}$ & $0.74^{\ddagger}$ & 0.08 & 0.15 & -0.22 \\
\hline & $\operatorname{Con} 350^{\circ} \mathrm{s}^{-1}$ & $0.71^{\ddagger}$ & -0.11 & 0.12 & 0.00 \\
\hline & $\operatorname{Ecc} 50^{\circ} s^{-1}$ & $0.76^{\ddagger}$ & 0.04 & 0.05 & -0.09 \\
\hline & $\operatorname{Ecc} 350^{\circ} \mathrm{s}^{-1}$ & $0.69^{\ddagger}$ & 0.01 & 0.09 & -0.10 \\
\hline & $T_{\text {con350 }} / T_{\text {isom }}$ & 0.27 & -0.16 & -0.09 & 0.24 \\
\hline
\end{tabular}

539 MHC, myosin heavy chain; BFlh, biceps femoris long head; Ecc, eccentric; Con, concentric;

$540{ }^{\dagger} P<0.01,{ }^{\ddagger} P<0.001$

541 
542 Table 3. Bivariate correlation coefficients between knee flexor explosive strength 543 characteristics, measured as time to specific (absolute and relative) torques, with hamstrings 544 muscle volume and biceps femoris long head composition.

\begin{tabular}{|c|c|c|c|c|c|c|c|}
\hline & & \multicolumn{6}{|c|}{ Explosive strength characteristics } \\
\hline & & \multicolumn{3}{|c|}{ Time to absolute torque } & \multicolumn{3}{|c|}{$\begin{array}{c}\text { Time to relative torque } \\
\text { (\%MVT) }\end{array}$} \\
\hline & & $10 \mathrm{Nm}$ & $50 \mathrm{Nm}$ & $90 \mathrm{Nm}$ & $15 \%$ & $45 \%$ & $75 \%$ \\
\hline Hamstrings & volume & & & & & & \\
\hline$\left(\mathrm{cm}^{3}\right)$ & & -0.10 & -0.43 & $-0.53^{*}$ & 0.01 & -0.16 & -0.24 \\
\hline MHC-I (\%) & & -0.09 & 0.14 & 0.06 & -0.01 & 0.14 & 0.24 \\
\hline MHC-IIA (\%) & & 0.01 & -0.06 & -0.15 & 0.03 & -0.05 & -0.05 \\
\hline MHC-IIX (\%) & & 0.08 & -0.09 & 0.08 & -0.02 & -0.09 & -0.20 \\
\hline
\end{tabular}

545 MHC, myosin heavy chain; MVT, maximal voluntary torque; * $P<0.05$ 